\title{
Ulinastatin enhances the therapeutic effect of intraperitoneal lavage on severe acute pancreatitis in rats
}

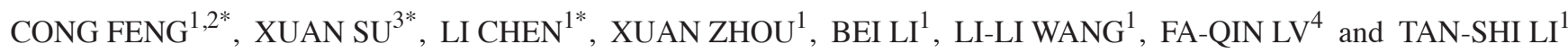 \\ ${ }^{1}$ Department of Emergency, General Hospital of the PLA, Beijing 100853; \\ ${ }^{2}$ The Li-Shi Road Out-Patient Department of General Hospital of the Second Artillery, Beijing 100045; \\ ${ }^{3}$ Department of Hepatology-Immunology, Beijing Youan Hospital of the Capital Medical University, Beijing 100069; \\ ${ }^{4}$ Department of Ultrasound, General Hospital of the PLA, Beijing 100853, P.R. China
}

Received May 19, 2014; Accepted December 17, 2014

DOI: $10.3892 / \mathrm{etm} .2015 .2334$

\begin{abstract}
The present study investigated the therapeutic effect of peritoneal lavage with ulinastatin on the outcome of rats with severe acute pancreatitis (SAP). A total of 110 male Wistar rats were randomly divided into the following groups: Control (C), SAP model (SAP), saline lavage (SL), intravenous ulinastatin (IU) and low-dose (LUL), medium-dose (MUL), high-dose (HUL) and ultrahigh-dose (UHUL) ulinastatin lavage. The treatments were performed immediately subsequent to the establishment of the SAP model. Intraperitoneal lavage with or without ulinastatin was performed for $3 \mathrm{~h}$. Survival time was recorded for $12 \mathrm{~h}$ and the median survival time was calculated. Histopathological analyses, and measurements of plasma amylase and lipase levels were performed. Blood $\mathrm{pH}$, lactic acid and base excess were also detected. The LUL, MUL, HUL, UHUL and IU groups showed an increase in the median survival time compared with the SAP group, with the maximal effect observed in the MUL group $(\mathrm{P}<0.01)$. The SL, MUL and IU groups showed a reduced activity of amylase and lipase compared with the SAP group. The SL $(\mathrm{P}<0.01)$ and the MUL groups $(\mathrm{P}<0.01)$ additionally showed a reduction in the lactic acid in arterial blood relative to the SAP group but the IU group did not. The MUL group showed greater improvements in $\mathrm{pH}$ $(\mathrm{P}<0.01)$ and base excess $(\mathrm{P}<0.01)$ versus the SAP group than the SL and IU groups. Furthermore the MUL group demonstrated a more marked reduction in the histological changes in
\end{abstract}

Correspondence to: Dr Tan-Shi Li, Department of Emergency, General Hospital of the PLA, 28 Fuxing Road, Haidian, Beijing 100853, P.R. China

E-mail: 1ts301@sohu.com

Dr Fa-Qin Lv, Department of Ultrasound, General Hospital of the PLA, 28 Fuxing Road, Haidian, Beijing 100853, P.R. China

E-mail:1vjin8912@163.com

${ }^{*}$ Contributed equally

Key words: intraperitoneal lavage, severe acute pancreatitis, ulinastatin necrosis, edema and inflammation compared with the SL and IU groups. Intraperitoneal lavage with ulinastatin significantly improves the prognosis of SAP in rats.

\section{Introduction}

Severe acute pancreatitis (SAP) is a serious systemic disease with high rates of mortality. Intraperitoneal lavage is a routine therapeutic regimen for SAP (1). Intraperitoneal lavage therapy with added protease inhibitors, such as camostate or glutaryl-trialanin-ethylamide, has been proved to improve survival in experimental SAP in several species (2-4). Ulinastatin is a purified glycoprotein from the urine of healthy adult males, which has a potent inhibitory effect on the activities of trypsin. Ulinastatin has been tested in numerous forms of acute pancreatitis and has been shown to exert significant therapeutic effects (5-7). Injection of ulinastatin, immediately after establishment of an SAP rat model, led to a significant decline in serum amylase levels, and a significant reduction in pathological changes in the pancreas (8). Ulinastatin may also efficiently interfere with SAP-associated acute lung injury through anti-inflammatory functions (9). Ulinastatin has a good effect on the recovery of blood pressure, heart rate, respiratory rate, bowel sound, serum amylase and urine amylase in patients with acute pancreatitis, which can prevent acute pancreatitis from worsening and reduce complications (10). However, peritoneal lavage with the addition of ulinastatin to lavage fluid has not been studied in SAP models. The theoretical advantage of ulinastatin is its antiprotease effects, which may protect the functions and the histopathology of the pancreas and other organs (8). The effect of intraperitoneal lavage with ulinastatin added to the lavage fluid on the outcome of taurocholate-induced SAP in rats was evaluated in the present study to provide a new experimental and theoretical basis for the treatment of SAP in the clinical setting.

\section{Materials and methods}

Experimental animals. A total of 110 healthy male Wistar rats that weighed $300 \pm 15 \mathrm{~g}$ were obtained from the Experimental Animal Center of the General Hospital of the PLA (Beijing, China). The experimental protocol was approved by the 
Ethics Committee for Animal Research from the General Hospital of the PLA and all experimental rats received humane care.

Reagents. Reagents were purchased from the following companies: Chloral hydrate (Shanghai Yingxin Industrial Co. Ltd., Shanghai, China); sodium taurocholate (Shanghai Hufeng Biotechnology Co. Ltd., Shanghai, China); ulinastatin (Guangdong Tianpu Biochemical Pharmaceutical Co. Ltd., Guangzhou, China); amylase and lipase assay kit (Shanghai Shifeng Biotechnology Co. Ltd., Shanghai, China).

Experimental groups. Rats were randomly divided into eight groups: i) Control ( $n=18$, group C), sham-operated without the induction of SAP, peritoneal lavage or intravenous injection, but with a catheter inserted; ii) SAP model ( $\mathrm{n}=18$, group SAP), induction of SAP without peritoneal lavage or intravenous injection but with a catheter inserted; iii) saline lavage $(n=18$, group SL), induction of SAP with saline lavage; iv) low-dose ulinastatin $(n=10$, group LUL), induction of SAP with $25 \mathrm{U} / \mathrm{ml}$ ulinastatin lavage; $v)$ medium-dose ulinastatin $(\mathrm{n}=18$, group MUL), induction of SAP with $62.5 \mathrm{U} / \mathrm{ml}$ ulinastatin lavage; vi) high-dose ulinastatin ( $\mathrm{n}=10$, group HUL), induction of SAP with $125 \mathrm{U} / \mathrm{ml}$ ulinastatin lavage; vii) ultrahigh-dose ulinastatin lavage ( $n=10$, group UHUL), induction of SAP with $250 \mathrm{U} / \mathrm{ml}$ ulinastatin lavage; viii) intravenous ulinastatin $(\mathrm{n}=18$, group IU), induction of SAP with $2,500 \mathrm{U} / 100 \mathrm{~g}$ intravenous ulinastatin and a catheter inserted but without peritoneal lavage.

Animal model. The rats were fasted for $12 \mathrm{~h}$ and had no access to water for $4 \mathrm{~h}$ prior to undergoing surgery. Rats were anesthetized with intraperitoneal injections of $10 \%$ chloral hydrate ( $3 \mathrm{ml} / \mathrm{kg}$ ). Following an incision into the abdomen, the distal region of the duodenal bile duct was clamped with injury-free metal clips, a syringe needle was inserted into the opening of the duodenal bile duct. Then, $5 \%$ sodium taurocholate (freshly prepared in saline solution, $0.6 \mathrm{ml}$ ) was retrogradely injected into the bile duct at a constant rate of $0.2 \mathrm{ml} / \mathrm{min}$ using an infusion pump (Zhejiang University Medical Instrument Co., Ltd., Hangzhou, China). After $5 \mathrm{~min}$, the needle and metal clips were removed. A consistently high mortality rate was produced ( $>80 \%$ within $12 \mathrm{~h}$ ). Group C was sham-operated without the induction of SAP.

Prior to closure of the abdomen, a silicon catheter (Catheter A; Shandong Weigao Group Medical Polymer Co., Ltd., Weihai, China) with five lateral outlets was placed adjacent to the pancreas and another silicon catheter (Catheter B; Shandong Weigao Group Medical Polymer Co., Ltd.) with five lateral outlets was placed in the pelvic cavity. The groups all accepted peritoneal catheter insertion.

Peritoneal lavage. Intraperitoneal lavage was performed immediately subsequent to the establishment of the SAP model in the SL, LUL, MUL, HUL and UHUL groups. Warmed $\left(37^{\circ} \mathrm{C}\right)$ lavage fluid was injected from catheter A at $80 \mathrm{ml} / \mathrm{h}$ for $15 \mathrm{~min}$ and catheter B was blocked. Catheter A was then blocked and fluid was allowed to flow out for 15 min from catheter B. Thus, each lavage procedure lasted $30 \mathrm{~min}$, and lavage was performed six times, for $3 \mathrm{~h}$ in total.
The input and output volumes were monitored. The lavage fluid consisted of saline solution with or without ulinastatin added at four concentrations: 25, 62.5, 125 and $250 \mathrm{U} / \mathrm{ml}$. Following lavage, catheters A and B were blocked and the rats were kept in single cages with free access to water but no solid food.

Intravenous ulinastatin. To compare the additional effects of peritoneal lavage with those of the intravenous administration of ulinastatin, the IU group received intravenous ulinastatin $2,500 \mathrm{U} / 100 \mathrm{~g}$ (freshly prepared in $0.15 \mathrm{ml}$ saline solution, approximately equal to the total dose of the MUL group) from the caudal vein immediately following SAP induction, but no lavage.

Assays and calculations. Survival time was recorded for $12 \mathrm{~h}$ and the median survival time for each group was calculated. Animals surviving to $12 \mathrm{~h}$ were anesthetized and sacrificed.

Rats in groups C, SAP, SL, MUL and IU ( $\mathrm{n}=8$ for each group) were sacrificed for histology and amylase and lipase measurements $3 \mathrm{~h}$ after the establishment of each model. Arterial blood was also collected into heparinized syringes from the abdominal aorta following general anesthesia and a second laparotomy. Blood $\mathrm{pH}$, lactic acid and base excess were detected using a blood gas analyzer (GEM 3000; Instrumentation Laboratory Company, Bedford, MA, USA). The activity of plasma amylase and lipase was determined using an automatic biochemical analyzer (Beckman Coulter-AU5800; Beckman Coulter, Indianapolis, IN, USA).

Pancreatic tissue for histology was fixed in formalin, subjected to conventional dehydration, embedded in paraffin and then sectioned into 5- $\mu \mathrm{m}$ sections, for subsequent staining by hematoxylin and eosin. Examination by light microscopy was performed by two professional pathologists using a double-blind method as to whether the section was from the treatment or the control groups. Three slices were randomly selected from each group; 10 high-power fields of vision were randomly selected for each slice and the extent of pancreatic tissue damage due to edema, inflammation and necrosis was evaluated as the histological parameters for grading the specific tissue damage to the pancreas. The pathological score for pancreatic tissue was calculated according to Rongione's standards as a reference with a minimum score of 0 and the highest score of 4 (11).

Statistical analysis. Data are expressed as mean \pm standard deviation. Statistical analyses were performed using the SPSS software package (version 19.0; IBM SPSS, Armonk, NY, USA). In the survival experiments, the survival time after the 12-h observation period was conducted by the Kaplan-Meier or Kruskal-Wallis $\mathrm{H}$ test. Analysis of variance or the Kruskal-Wallis $\mathrm{H}$ test was used for comparisons of biochemical data among all groups. $\mathrm{P}<0.05$ was considered to indicate a statistically significant difference.

\section{Results}

Effect of intraperitoneal lavage with different ulinastatin concentrations on the survival time. The rats in group C remained alive after $12 \mathrm{~h}$. The median survival time of the SAP group was $4.83 \mathrm{~h}$. The SL group did not show a prolonged 
A

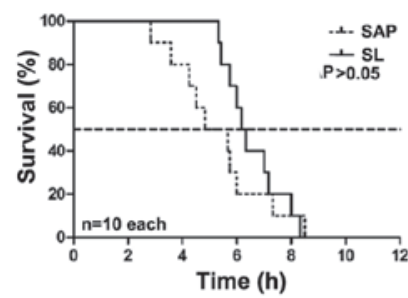

B

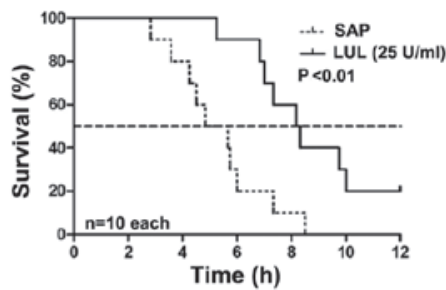

D

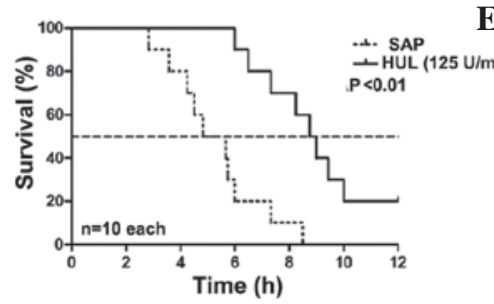

$\mathbf{E}$

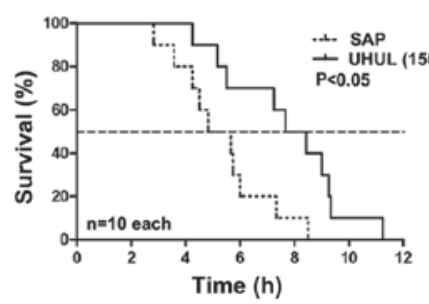

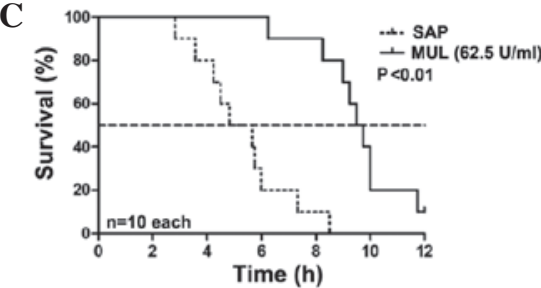

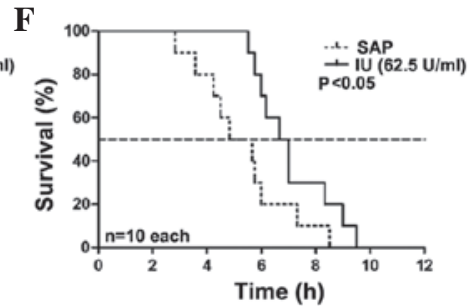

Figure 1. Effect of peritoneal lavage with saline and different concentrations of ulinastatin on the survival time compared with the SAP group. (A) SL; (B) LUL; (C) MUL; (D) HUL; (E) UHUL; and (F) IU. Treatments were performed during the first $3 \mathrm{~h}$ after SAP was induced in the rats, with taurocholate. SL did not show any beneficial effect $(\mathrm{P}>0.05)$ but median survival time was significantly improved by all concentrations of ulinastatin. The maximal effect was observed in the MUL group with $62.5 \mathrm{U} / \mathrm{ml}$ ulinastatin $(\mathrm{P}<0.01)$. IU also showed a beneficial effect $(\mathrm{P}<0.05)$ but less so than the MUL group. SAP, severe acute pancreatitis; SL, saline lavage; LUL, low-dose ulinastatin lavage; MUL, medium-dose ulinastatin lavage; HUL, high-dose ulinastatin lavage; UHUL, ultrahigh-dose ulinastatin lavage; IU, intravenous ulinastatin without peritoneal lavage.

A

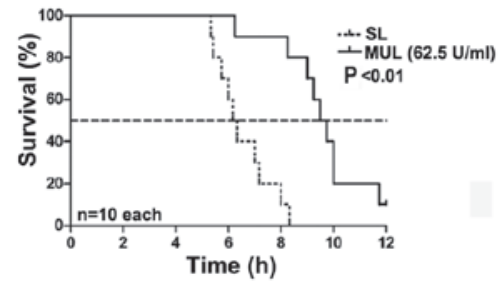

B

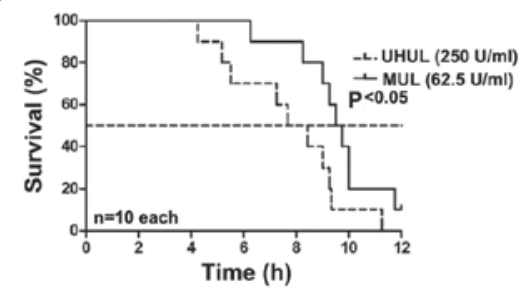

C

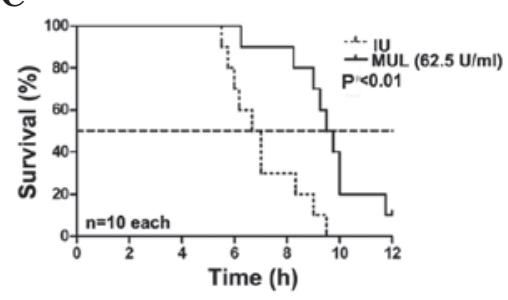

Figure 2. Effect of ulinastatin lavage at $62.5 \mathrm{U} / \mathrm{ml}$ on survival time. (A) MUL versus SL. (B) MUL versus UHUL. (C) MUL versus IU. At concentrations of $62.5 \mathrm{U} / \mathrm{ml}$ (MUL group) the effect of ulinastatin lavage was superior to that observed with SL $(\mathrm{P}<0.01)$. The therapeutic effects were attenuated at $250 \mathrm{U} / \mathrm{ml}$ (UHUL group) compared with the MUL group $(\mathrm{P}<0.05)$. The IU $(2,500 \mathrm{U} / 100 \mathrm{~g})$ also showed a beneficial effect $(\mathrm{P}<0.01)$ on the median survival time, but less so than the MUL. MUL, medium-dose ulinastatin lavage; UHUL, ultrahigh-dose ulinastatin lavage; IU, intravenous ulinastatin without lavage; SL, saline lavage.

median survival time compared with the SAP group (6.17 vs. $4.83 \mathrm{~h}$; P>0.05, Fig. 1); however, $25 \mathrm{U} / \mathrm{ml}$ ulinastatin added to the lavage fluid (LUL group) began to significantly increase the median survival time ( 8.17 vs. $4.83 \mathrm{~h} ; \mathrm{P}<0.01$, Fig. 1$)$, and a maximal effect was exerted with the addition of $62.5 \mathrm{U} / \mathrm{ml}$ ulinastatin to the lavage fluid (MUL group) (MUL versus SAP, 9.50 vs. 4.83 h; $\mathrm{P}<0.01$, Fig. 1) (MUL versus SL, 9.50 vs. 6.17 h; $\mathrm{P}<0.01$, Fig. 2). A concentration of $125 \mathrm{U} / \mathrm{ml}$ ulinastatin (HUL group) caused similar results to the MUL group when compared with the SAP group (8.75 vs. $4.83 \mathrm{~h}$; P<0.01, Fig. 1). The therapeutic effects were attenuated at a concentration of $250 \mathrm{U} / \mathrm{ml}$ (UHUL group) compared with the MUL group (7.67 vs. $9.50 \mathrm{~h}$; $\mathrm{P}<0.05$, Fig. 2) but the UHUL group also showed a significantly increased median survival time compared with the SAP group (7.67 vs. $4.83 \mathrm{~h}$; $\mathrm{P}<0.05$, Fig. 1). Intravenous ulinastatin of 2,500 U/100g (IU group) showed a beneficial effect on the median survival time compared with the SAP group (6.67 vs. $4.83 \mathrm{~h} ; \mathrm{P}<0.05$, Fig. 1) but a diminished effect when compared with the MUL group (6.67 vs. $9.50 \mathrm{~h} ; \mathrm{P}<0.01$, Fig. 2). The results are summarized in Table I.

Effect of lavage with ulinastatin on biochemical parameters. The activity of amylase and lipase in the plasma of the SAP group was significantly increased in comparison with that in group C. The SL, MUL and IU groups all showed significantly reduced amylase and lipase activity compared with the SAP group, with the treatment in the MUL group exerting the maximal effect.

The levels of lactic acid in the arterial blood of the SAP group were significantly increased in comparison with those in group C. The lactic acid levels were reduced in the SL and MUL groups when compared with those in the SAP group but no significant difference was observed between the IU and SAP groups. The maximal effect was observed in the MUL group. The MUL and SL group treatments also resulted in an improved $\mathrm{pH}$ and base excess, as compared with the SAP group, but the IU group treatments did not. The IU group treatment did not affect blood $\mathrm{pH}$, lactic acid or base excess in arterial blood compared with the SAP group. The results of the effects of lavage with ulinastatin on biochemical parameters are summarized in Table II.

Effect of lavage with ulinastatin on histopathological features of pancreatic tissue. The histological changes $3 \mathrm{~h}$ after the onset of SAP were significantly reduced in the MUL group when compared with those in the SAP group with regard to necrosis, edema and inflammation. The IU group also showed improved 
Table I. Comparison of survival time following the induction of SAP in rats.

\begin{tabular}{|c|c|c|c|c|c|c|}
\hline \multirow[b]{2}{*}{ Group } & \multirow[b]{2}{*}{$\mathrm{n}$} & \multirow[b]{2}{*}{$\begin{array}{l}\text { Median survival } \\
\text { time }(\mathrm{h})\end{array}$} & \multirow[b]{2}{*}{$\begin{array}{l}\text { Interquartile } \\
\text { range }(\mathrm{h})\end{array}$} & \multicolumn{3}{|c|}{ P-value } \\
\hline & & & & $\begin{array}{l}\text { Compared with } \\
\text { the SAP group }\end{array}$ & $\begin{array}{l}\text { Compared with } \\
\text { the IU group }\end{array}$ & $\begin{array}{l}\text { Compared with } \\
\text { the SL group }\end{array}$ \\
\hline SAP & 10 & 4.83 & $2.83-8.50$ & & & \\
\hline IU & 10 & 6.67 & $5.50-9.50$ & $0.044^{\mathrm{a}}$ & & \\
\hline SL & 10 & 6.17 & $5.33-8.33$ & 0.370 & 0.267 & \\
\hline LUL & 10 & 8.17 & $5.25-12.00$ & $0.003^{\mathrm{b}}$ & $0.046^{\mathrm{a}}$ & $0.011^{\mathrm{a}}$ \\
\hline MUL & 10 & 9.50 & $6.25-12.00$ & $0.000^{\mathrm{b}}$ & $0.002^{\mathrm{b}}$ & $0.000^{\mathrm{b}}$ \\
\hline HUL & 10 & 8.75 & $6.00-12.00$ & $0.000^{\mathrm{b}}$ & $0.040^{\mathrm{a}}$ & $0.001^{\mathrm{b}}$ \\
\hline UHUL & 10 & 7.67 & $4.25-11.25$ & $0.015^{\mathrm{a}}$ & 0.352 & $0.034^{\mathrm{a}}$ \\
\hline
\end{tabular}

${ }^{a} \mathrm{P}<0.05 ;{ }^{b} \mathrm{P}<0.01$. SAP, severe acute pancreatitis; IU, intravenous ulinastatin $(2,500 \mathrm{U} / 100 \mathrm{~g})$; SL, saline lavage; LUL, low-dose ulinastatin lavage (25 U/ml); MUL, medium-dose ulinastatin lavage (62.5 U/ml); HUL, high-dose ulinastatin lavage (125 U/ml); UHUL, ultra-high-dose ulinastatin lavage $(250 \mathrm{U} / \mathrm{ml})$.

Table II. Effect of lavage with the addition of $62.5 \mathrm{U} / \mathrm{ml}$ ulinastatin (MUL) to lavage fluid on amylase and lipase activity in plasma, arterial blood gases and the histological degree of pancreatitis.

\begin{tabular}{|c|c|c|c|c|c|}
\hline Parameter & Control & SAP & SL & MUL & IU \\
\hline \multicolumn{6}{|l|}{ Plasma } \\
\hline Amylase, U/1 & $1831.1(437.6)^{\mathrm{a}}$ & $10172.0(1286.0)$ & $5651.8(1293.9)^{\mathrm{a}}$ & $3426.8(484.4)^{\mathrm{a}}$ & $6275.1(988.5)^{\mathrm{a}}$ \\
\hline Lipase, U/1 & $42.8(21.4)^{\mathrm{a}}$ & $3789.4(496.5)$ & $2476.9(618.5)^{\mathrm{a}}$ & $1398.8(384.2)^{\mathrm{a}}$ & $2478.5(385.2)^{\mathrm{a}}$ \\
\hline \multicolumn{6}{|l|}{ Arterial blood } \\
\hline $\mathrm{pH}$ & $7.26(0.04)^{\mathrm{a}}$ & $7.04(0.06)$ & $7.07(0.06)$ & $7.18(0.06)^{\mathrm{a}}$ & $7.05(0.06)$ \\
\hline Lactic acid, mmol/1 & $3.40(1.07)^{\mathrm{a}}$ & $5.45(1.04)$ & $3.62(0.59)^{\mathrm{a}}$ & $3.33(0.74)^{\mathrm{a}}$ & $5.04(1.01)$ \\
\hline Base excess, mmol/1 & $6.83(1.33)^{\mathrm{a}}$ & $18.99(1.35)$ & $17.88(1.00)^{\mathrm{b}}$ & $16.74(1.07)^{\mathrm{a}}$ & $18.61(1.25)$ \\
\hline \multicolumn{6}{|l|}{ Histological gradings $\mathrm{s}^{\mathrm{c}}$} \\
\hline Necrosis & $0^{\mathrm{a}}$ & 3 & 3 & $2^{\mathrm{a}}$ & 3 \\
\hline Edema & $0^{\mathrm{a}}$ & 3 & $2-3^{b}$ & $1^{\mathrm{a}}$ & $2^{\mathrm{a}}$ \\
\hline Inflammation & $0^{\mathrm{a}}$ & 3 & 3 & $1-2^{\mathrm{a}}$ & $1-2^{\mathrm{a}}$ \\
\hline
\end{tabular}

${ }^{\mathrm{a}} \mathrm{P}<0.01$ and ${ }^{\mathrm{b}} \mathrm{P}<0.05$ vs. the SAP group; ${ }^{\mathrm{c}}$ calculated according to Rongione's standards. Data for plasma and arterial blood are presented as the mean (standard deviation). SAP, severe acute pancreatitis; SL, saline lavage; MUL, medium-dose ulinastatin lavage; IU, intravenous ulinastatin.

edema and inflammation scores compared with those for the SAP group but no difference was observed in necrosis. The SL group showed improved edema results but no effect was noted on necrosis and inflammation. The results are also summarized in Table II.

\section{Discussion}

Peritoneal antiprotease lavage therapy has been the subject of experimental and clinical research in pancreatitis for $>40$ years. It has been reported that when pancreatic enzymes are released into the peritoneal exudate they may lead to hypotension and mortality so that peritoneal lavage becomes necessary, and antiprotease therapy should be directed locally into the peritoneal cavity for the greatest therapeutic effect (12-14). Certain studies have shown that peritoneal lavage with the addition of a protease inhibitor to the lavage fluid improves the mortality in experimental SAP $(1,4)$. Although some studies in humans have not shown a significant improvement in survival with peritoneal antiprotease lavage therapy, it was reported that this treatment could counteract the development of pancreatic necrosis for patients with SAP and reduce the need for surgical intervention $(12,15,16)$.

Ulinastatin is a purified glycoprotein from healthy adult male urine, and numerous studies suggest that it has a significant therapeutic effect on SAP (17-19). In previous years, ulinastatin has been widely used for the treatment of patients with SAP in Asia. The therapeutic effect is greater using an arterial infusion catheter than that with an intravenous injection, as only a small amount of the intravenously administered ulinastatin reaches the pancreas $(20-22)$. To the best of our knowledge, the experimental study of peritoneal lavage 
with the addition of ulinastatin in SAP has not been reported, and there has been no commitment to a clinical study.

The present study is the first to evaluate the effect of peritoneal lavage with the addition of ulinastatin to the lavage fluid in SAP rats. The retrograde injection of $5 \%$ sodium taurocholate (freshly prepared in saline solution, $0.6 \mathrm{ml}$ ) into the pancreatic duct at the rate of $0.2 \mathrm{ml} / \mathrm{min}$ produced an SAP model with a consistently high mortality rate (>80\% within $12 \mathrm{~h}$ ) (4). This high-mortality SAP model was selected in order that the study under investigation would be comparable to a life-threatening pancreatitis in humans.

Peritoneal lavage with ulinastatin added to the lavage fluid significantly improved the median survival time. In the MUL group, the enzyme activity of amylase and lipase, arterial blood lactic acid, blood $\mathrm{pH}$ and base excess, and the histological degree of the pancreatitis were significantly improved compared with the SAP group. Furthermore, the MUL group treatment exerted superior effects to the treatments in the different concentration groups. The therapeutic effects of intraperitoneal lavage with 62.5 U/ml ulinastatin added to the lavage fluid on the outcome of SAP are better than those of lavage alone and intravenous interventions. The microcirculation could also be significantly improved by intraperitoneal lavage with ulinastatin, which resulted in marked reductions in the lactic acid levels and base excess.

The beneficial effects of lavage with ulinastatin are associated with a reduction in the severity of pancreatitis and an improved circulation as estimated by histology, enzyme activity in the blood plasma and blood gases; thus, the improvement in survival time by the intraperitoneal lavage with ulinastatin may stem from combinational effects, including local and systemic effects.

In the MUL group, the survival rate at $12 \mathrm{~h}$ (1 out of 10) was not significantly better than that of the SAP group (0 out of 10). The short-term effect of peritoneal lavage may be one of the reasons. It has been reported that early and extended peritoneal lavage may be a useful therapy in the management of SAP (23); therefore, further studies are required to show that the addition of ulinastatin into the peritoneal lavage fluid further improves the survival rate and multiple organ function in SAP when the procedure of peritoneal lavage is prolonged. The combinational effect of intravenous ulinastatin and peritoneal lavage with ulinastatin may have superior therapeutic effects; this could be the subject of further studies.

\section{Acknowledgements}

The authors are grateful to Professor Chong-Hiu Li (Department of Hepatobiliary Surgery, General Hospital of the PLA) for her recommendations.

\section{References}

1. Niederau C, Crass RA, Silver G, Ferrell LD and Grendell JH: Therapeutic regimens in acute experimental hemorrhagic pancreatitis. Effects of hydration, oxygenation, peritoneal lavage, and a potent protease inhibitor. Gastroenterology 95: 1648-1657, 1988.
2. Wilson $\mathrm{C}$ and Imrie $\mathrm{CW}$ : Effective intraperitoneal antiprotease therapy for taurocholate-induced pancreatitis in rats. Br J Surg 77: 1252-1255, 1990.

3. Fric P, Slabý J, Kasafírek E, Kocna P and Marek J: Effective peritoneal therapy of acute pancreatitis in the rat with glutaryl-trialanin-ethylamide: a novel inhibitor of pancreatic elastase. Gut 33: 701-706, 1992.

4. Leonhardt U, Seidensticker F, Fussek M, Stöckmann F and Creutzfeldt W: Camostate (FOY-305) improves the therapeutic effect of peritoneal lavage on taurocholate induced pancreatitis. Gut 31: 934-937, 1990.

5. Ohnishi H, Kosuzume H, Ashida Y, Kato K and Honjo I: Effects of urinary trypsin inhibitor on pancreatic enzymes and experimental acute pancreatitis. Dig Dis Sci 29: 26-32, 1984.

6. Tani S, Otsuki M, Itoh $\mathrm{H}$ et al: The protective effect of the trypsin inhibitor urinastatin on cerulein-induced acute pancreatitis in rats. Pancreas 3: 471-476, 1988.

7. Inoue K, Takano H, Shimada A et al: Urinary trypsin inhibitor protects against systemic inflammation induced by lipopolysaccharide. Mol Pharmacol 67: 673-680, 2005.

8. Li F, Zhang H, Xu KY, Wei Q and Zhou GX: Role of the chemokine fractalkine in a rat model of acute necrotizing pancreatitis and the interventional effect of ulinastatin. Arch Iran Med 16: 83-87, 2013.

9. Inoue K, Takano H, Shimada A, et al: Urinary trypsin inhibitor protects against systemic inflammation induced by lipopolysaccharide. Mol Pharmacol 67: 673-680, 2005.

10. Li HM, Feng QX, Dou KF, et al: Treatment of acute pancreatitis with ulinastatin: a report of 72 cases. Xi Bei Guo Fang Yi Xue Za Zhi 23: 183-185, 2002 (In Chinese).

11. Rongione AJ, Kusske AM, Kwan K, et al: Interleukin 10 reduces the severity of acute pancreatitis in rats. Gastroenterology 112: 960-967, 1997.

12. Balldin G, Borgström A, Genell S and Ohlsson K: The effect of peritoneal lavage and aprotinin in the treatment of severe acute pancreatitis. Res Exp Med (Berl) 183: 203-213, 1983.

13. Ohlsson $\mathrm{K}$ and Tegner $\mathrm{H}$ : Experimental pancreatitis in the dog. Demonstration of trypsin in ascitic fluid, lymph and plasma. Scand J Gastroenterol 8: 129-133, 1973.

14. Bhatia M, Wong FL, Cao Y et al: Pathophysiology of acute pancreatitis. Pancreatology 5: 132-144, 2005.

15. Berling R, Borgström A and Ohlsson K: Peritoneal lavage with aprotinin in patients with severe acute pancreatitis. Effects on plasma and peritoneal levels of trypsin and leukocyte proteases and their major inhibitors. Int J Pancreatol 24: 9-17, 1998.

16. Dong Z, Petrov MS, Xu J, et al: Peritoneal lavage for severe acute pancreatitis: a systematic review of randomised trials. World J Surg 34: 2103-2108, 2010.

17. Hirano $T$ and Manabe T: Human urinary trypsin inhibitor, urinastatin, prevents pancreatic injuries induced by pancreaticobiliary duct obstruction with cerulein stimulation and systemic hypotension in the rat. Arch Surg 128: 1322-1329, 1993.

18. Wang G, Wen J, Wilbur RR, et al: The effect of somatostatin, ulinastatin and Salvia miltiorrhiza on severe acute pancreatitis treatment. Am J Med Sci 346: 371-376, 2013.

19. Wallner G, Solecki M, Ziemiakowicz R, et al: Morphological changes of the pancreas in course of acute pancreatitis during treatment with Ulinastatin. Pol Przegl Chir 85: 114-122, 2013.

20. Keck T, Balcom JH, Antoniu BA, et al: Regional effects of nafamostat, a novel potent protease and complement inhibitor, on severe necrotizing pancreatitis. Surgery 130: 175-181, 2001

21. Watanabe S: Acute pancreatitis: overview of medical aspects. Pancreas 16: 307-311, 1998.

22. Matsukawa H, Hara A, Ito $\mathrm{T}$ et al: Continuous arterial infusion of protease inhibitor with supplementary therapy for the patients with severe acute pancreatitis - clinical effect of arterial injection of ulinastatin. Nihon Shokakibyo Gakkai Zasshi 95: 1229-1234, 1998 (In Japanese).

23. Botoi G and Andercou A: Early and prolonged peritoneal lavage with laparoscopy in severe acute pancreatitis. Chirurgia (Bucur) 104: 49-53, 2009 (In Romanian). 